\title{
Investigation into the Future of RFID in Biomedical Applications
}

\author{
Lucas Ricciardi $^{a, b}$, Inke Pitz ${ }^{a, b, c}$, Said F. Al-Sarawi ${ }^{a, b}$, Vijay Varadan $^{d}$ and Derek Abbott ${ }^{a, b}$ \\ ${ }^{a}$ Centre of Biomedical Engineering, The University of Adelaide, SA 5005, Australia \\ ${ }^{b}$ Department of Electrical and Electronic Engineering, The University of Adelaide, SA 5005, \\ Australia \\ ${ }^{c}$ Department of Biomedical Engineering, University of Applied Sciences, 45877 Gelsenkirchen, \\ Germany \\ ${ }^{d}$ Research Center for the Engineering of Electronic and Acoustic Materials, Pennsylvania \\ State University, University Park, PA 16802, USA
}

\begin{abstract}
This paper focuses on the future of Radio Frequency Identification (RFID) systems in biomedical applications. It discusses current technology, restrictions and applications and also illustrates possible future development. We mainly focus on actuator devices and introduce some of the concepts for RFID sensors. Basically, Radio Frequency Identification is a technology that is analogous to the working principle of magnetic bar code systems. Our proposed solution is to exploit an exciting new development in making circuits on polymers without the need of battery power. This solution exploits the principle of a Surface Acoustic Wave (SAW) device on a polymer substrate. The SAW device is a set of interdigitated conducting fingers on the polymer substrate. If an appropriate RF signal is sent to the device, the fingers act as microantennas that pick up the signal, and this energy is then converted into acoustic waves that travel across the surface of the polymer substrate. Being a flexible polymer, the acoustic waves cause stress that can either contract or stretch the material. In our case we want to use RFID technology for biomedical applications. We want to use this technology for devices, which are implanted in the human body and controlled or observed by a radio frequency signal.
\end{abstract}

Keywords: RFID, SAW Devices, Coding, Microvalve

\section{INTRODUCTION}

Of the few current implantable devices such as pacemakers, very few are passive (they do not require an internal power source, such as batteries). A battery usually makes these devices relatively large and they only function for the lifetime of the battery. With RFID technology, passive transponders can be powered by RF energy, which means that devices can be significantly smaller and have much longer life spans. The main application for RFID in biomedicine involves inserting a device into small spaces in the body such as veins or in the case of fertility control, the vas deferens, which is only $400 \mu \mathrm{m}$ in diameter.

Since RFID devices can be used in many different environments, they have enormous potential for product development in the biomedicine industry. Some of the proposed applications are:

1) Actuator devices: Nano-litre drug delivery, male fertility control, peristaltic pumps, artificial valves

2) Sensor Devices: Heart valve monitoring, transponder developments.

Today, mainstream integrated circuits tend to use silicon as the substrate material. However, there is now a new growing field in the area of polymer electronics. This field is moving rapidly and researchers have successfully fabricated transistors on a polymer substrate. ${ }^{1}$ This new paradigm has the advantage that circuits can be flexible and lightweight. Also the material parameters of a polymer can be more finely tuned than in silicon. ${ }^{1}$ A further advantage is that polymer materials are more bio-friendly for implantation in animals or the human body - this is attractive for a host of applications from artificial valves to pacemakers. Polymer materials can be flexed and textured, whereas silicon is hard and brittle. The active circuitry on a polymer can be elegantly encapsulated and isolated from body fluids and tissues. 
There are many devices that already have been developed using RFID technology, for example in the case of nano-litre drug delivery. One example consists is a small enclosed capsule made of biomaterial or plastic. Inside the capsule are a silicon chip and an antenna that control the release of the drug from a small reserve within. The main problem with a device such as this is that the valves that release the drug are often sacrificial, ${ }^{2-4}$ which means that the devices have a limited life. Another major drawback is the fact that different sized valves are needed to release differing amounts of the drug. A further problem that current devices have is the fact that they rely heavily on MEMS's (micro-electromechanical systems) technology, which is not reliable enough for in vivo applications. Because of their mechanical nature that there is often significant wearing of moving parts and the problem of stiction, which both lead to failure.

\section{POLYMERS IN BIOMEDICAL RFID}

Biomedical RFID technology exploits the working principle of the piezoelectric effect, which is defined as a change in electric polarization resulting from a change in applied stress, which is usually referred to as the direct piezoelectric effect. A piezoelectric material has the characteristic of being elastic and generating an electric field when a force is applied to it and vice versa. Wave propagation on the surface of the piezoelectric material can thus be generated and detected by interdigital transducers (IDTs) on the surface of the material.

Basically, we may think of a SAW device as consisting of a solid substrate with an input and output transducer. Because of this, Interdigital Transducers (IDTs) provide a practical method for generating and detecting surface acoustic waves. The input transducer converts the incoming signal by the inverse piezoelectric effect into acoustic waves, which propagate along the planar surface of the solid. At the output transducer, the SAWs are reconverted to an electrical signal. The basic principle of a wireless SAW ID-tag is shown in Figure 1.

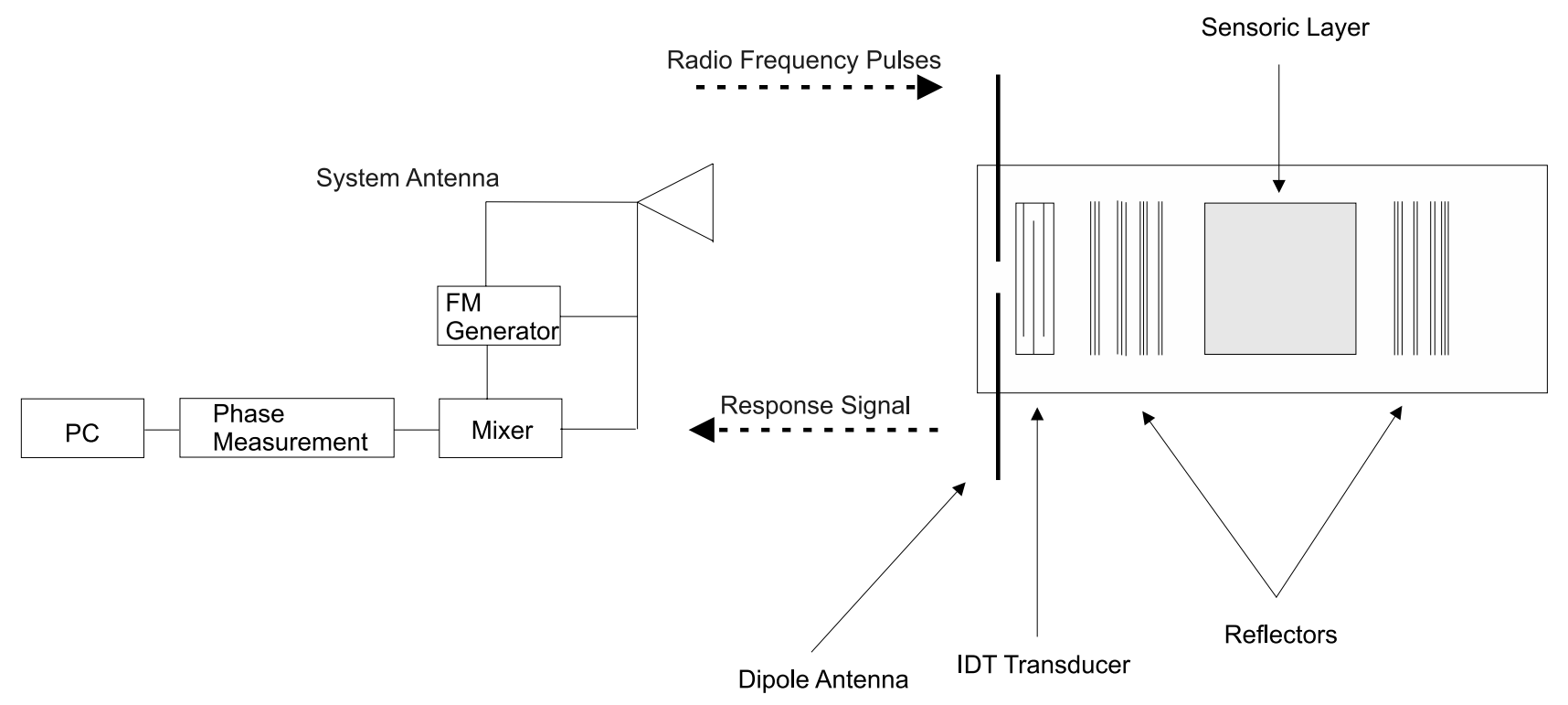

Figure 1. This figure shows the principle of a wireless SAW ID-tag which uses reflectors on its surface instead of an output IDT to reflect the SAWs back to the input IDT which is directly connected to the antenna. This antenna is able to receive as well as to transmit RF pulses. The reader/transmitter station with its system antenna are also shown. The reading system has a linear Frequency Modulated (FM) signal generator and the FM signals are transmitted by the system antenna.

As an alternative to the use of a two part SAW device, the receiving section can be replaced by a series of reflectors and the output Identification Device (ID-tag) can be directly connected to a microstrip antenna to receive and transmit $\mathrm{RF}$ signals. The transmitted $\mathrm{RF}$ signal is received by the IDT antenna and transformed into a propagating surface acoustic wave. The response signals form a sequence of short pulses in accordance with the number and position of the reflectors on the crystal surface. The time delay of every partial response 
pulse depends on the SAW propagation velocity, which is affected by several physical quantities (temperature, pressure and strain) and the distance between the IDT and the reflectors. ${ }^{5}$ The response signal is reflected to the IDT and transduced back into electromagnetic waves. These waves are radiated by the antenna and received and evaluated by the reader station. So it is possible to get information about the localization of the device as well as the ability to control its function. The basic structure and the working principle of an IDT are shown in Figure 2 .

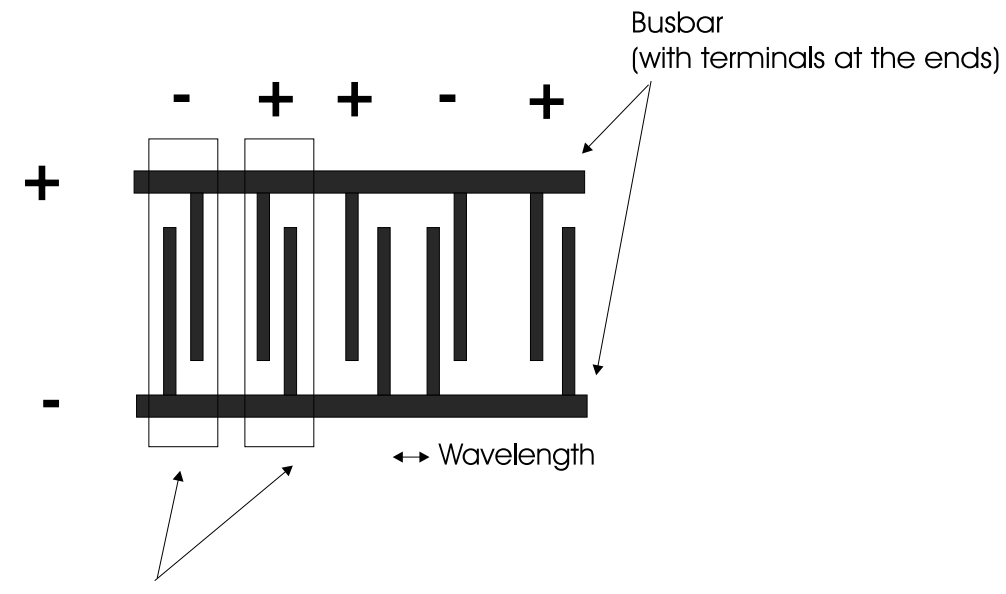

Finger pairs 1 and 2

Figure 2. This figure shows the basic structure of an IDT and how it works. When a voltage received from the antenna, which is directly connected to the IDT, this voltage is applied to the terminals of the transducer and creates a potential difference across the busbar. Each pair of fingers can be regarded as a capacitor. Hence the potential difference creates charge on each finger which is proportional to the applied voltage. The charge created causes a surface acoustic wave to be generated as a result of the piezoelectric effect. If we consider the first finger pair, the charge on the first finger creates a positive acoustic pulse and the charge on the second finger creates a negative pulse hence creating an overall negative pulse. It can be seen that the second finger pair is connected to the busbar with opposite sign to the first pair, so will generate an overall positive pulse. It is clearly apparent that by ordering the fingers in certain ways we can affect the creation of acoustic pulses. This effect is exploited in coding the devices as will be explained in later sections.

It is clearly apparent the importance of having a device that can be encoded if successful in vivo applications are to become a reality. With today's technology we can achieve encoding of SAW devices by designing the IDT's fingers in a specific order. By designing the IDT fingers in such a way so that their polarity and orientation is related to the input code, we can ensure that only a correct acoustic wave propagates. This can be seen in Figure 1. When a negative RF pulse is injected into the left most finger pair (pair 1), it excites a positive acoustic wave. We can see that the generated wave is opposite in sign to the injected pulse. This is due to the fact that the negative pulse injected into a negative finger pair cancels to be positive, for this reason we need the input code to be the equivalent to the finger pair configurations. The acoustic wave generated at the first finger pair will reach the receiving transducer at the same time as the wave from the second finger pair. Since these will be of the same sign and phase they will constructively interact to increase the size of the wave. From this we can see that if the incorrect code is input to the system the generated pulses will destructively interact. Hence by increasing the number of finger pairs we can increase the number of possible codes, which makes it more secure for the patient (because that makes it harder to manipulate or abuse the code). For example if the code $(+-$ ++- ) is input into the IDT shown in Figure 1 (with the right most pulse first) each pulse would generate a positive SAW that will arrive at the receive transducer and constructively interact. Because of that it is possible to produce a voltage that is significant enough to cause actuation. ${ }^{5}$

Given that most IDT devices have the restriction of finger widths being $\lambda / 4$ and finger pairs must be $\lambda$ apart, so that constructive interference occurs, we can use the equation

$$
\lambda=V / f,
$$


where $V$ is the velocity of the acoustic wave and $f$ is the received transmission frequency, to design an IDT to receive any required frequency. ${ }^{6}$

For example, if $V=3500 \mathrm{~m} / \mathrm{s}$ as for Lithium Niobate and $f=900 \mathrm{MHz}$, the finger pairs must be $3.8 \mu \mathrm{m}$ apart and the finger widths must be $0.9 \mu \mathrm{m}$ wide. From these fundamental restrictions we can calculate the number of finger pairs we can have, for a given valve size.

It should be noted that the fewer fingers an IDT has, the less precision it has in responding to a coded signal and the wider the bandwidth that will excite a surface acoustic wave. ${ }^{6}$ The diameter the human vas deferens is $400 \mu \mathrm{m}$, hence for male fertility control applications this limits the total size of the valve, and thus the IDT would have to be smaller than this. That means a code length of 64 bit will require an IDT of $243 \mu \mathrm{m}$. Further research is needed in this area to determine optimal input code lengths based on required actuation voltage for a particular device and the proposed application.

Sensor devices work by reflecting back the SAW to the IDT for retransmission. This is achieved by using reflective plates on the polymer surface that return the SAW to the IDT. These reflectors coupled with the attenuation through the polymer enforce a constraint on the maximum size of reply code to 32 bits. Any increase beyond this length results in the reflected SAW response being too small to be retransmitted. ${ }^{5}$

One possible way to use the resulting device as a valve, is to cut a slit into the polymer - as a SAW propagates through the substrate the slit will be periodically stretched and hence we have an oscillating aperture. When a fluid passes through the oscillating aperture its flow is restricted to a degree depending on its oscillation frequency. Hence when a SAW is generated by the IDT with a frequency of $900 \mathrm{MHz}$, the flow will be severely restricted. However this problem can be overcome to a degree, by choosing a dielectric film for the polymer that has a high dispersive effect. This works by dispersing the travelling acoustic wave as it propagates along the polymer substrate. With current technology the wave can be transformed down to a frequency of $100 \mathrm{~Hz}$, which only restricts the flow by a factor of 4 , for a $400 \mu \mathrm{m}$ aperture.

\section{POLYMER DEVICES FOR BIOMEDICINE}

As can be seen in Figure 3 the polymer device could be used as an RF controlled microvalve so that when the RF energy excites the SAW the polymer flexes and allows the valve to open. This type of device has applications for people with heart conditions or even varicose veins, where the valve inside the vein no longer works. ${ }^{7}$

When the surface acoustic waves cause stresses in the polymer substrate, the resulting deflection can be in the order of microns, ${ }^{8}$ suggesting an ideal novel application would be a microvalve. Essentially, a small hole in the polymer substrate can be stretched open wider or contracted by application of the RF signal on a SAW-on-polymer device. By sandwiching a number of these microvalves together, and by operating them in different phases, a peristaltic micropump could be created - this is very useful in cases where a fluid needs to be "pushed" through the orifice. Alternatively, the SAW could be used to actuate a V-groove valve arrangement (also called a "check valve"). A further possibility is for passive venous valves (such as in artificial heart valves) to use the polymer device for monitoring the open/shut status of the valve, rather than for actuation. A host of lucrative applications from valves for electronic fertility control to micropumps for nanolitre drug delivery become foreseeable. The basic structure of the V-groove microvalve is shown in Figure 3.

Three different polymer layers are utilized to create the valve:

1. UV curable structural polymer to form the body of the valve

2. a sacrificial polymer that can be etched away to leave cavities in the resulting 3D structure

3. a multifunctional polymer that has piezoelectrical properties that can act as either a SAW sensor or actuator

The structural polymer, UV 001, is from HVS Technologies. It is a UV curable polymer with urethene acrylate, epoxy acrylate and acryloxysilane as the main ingredients. Its low viscosity allows easy processing through automatic equipment or manual methods without the addition of solvents or heat to reduce the viscosity. It also complies with all VOC regulations. It has excellent flexibility and resistance to fungus, solvents, water and chemicals. The sacrificial polymer is essentially an acrylic resin containing $50 \%$ silica. This composition 


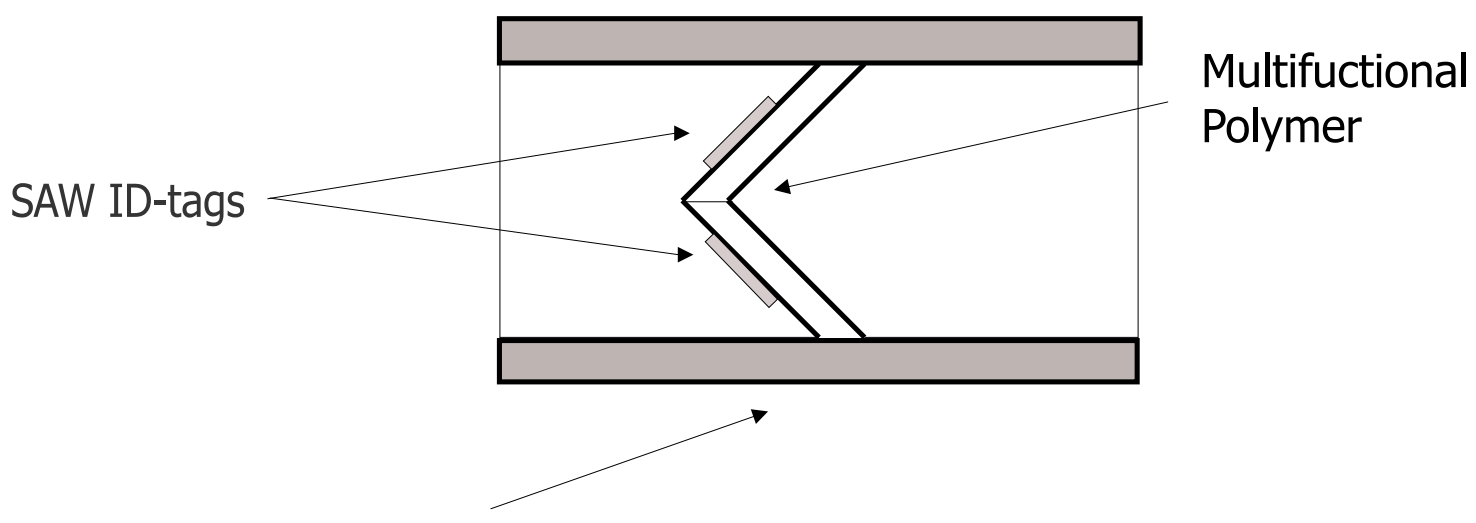

Structural Layer

Figure 3. This figure shows the basic structure of a V-groove microvalve enhanced by SAW tags. It can be a passive valve or an active one. The passive microvalve can be opened or closed by the pressure of the surrounding fluid depending on the direction of the fluid stream. In the case of a passive microvalve the SAW ID-tag electrodes have only the function of monitoring the status of the valve. That means that they are sending an RF signal to a reader station to give information about whether the valve is open or closed and where it is localized. But the microvalve can also be used as an active one. In this case the SAW ID-tag electrodes work as actuators stretching open the polymer flaps with a cantilever action.

can be dissolved with $2 \mathrm{Mol} / \mathrm{L}$ caustic soda at $80^{\circ} \mathrm{C}$. The multifunctional layer consists of nanoceramic particles attached by chemical bonding as side groups on a polymer backbone. The concept is a backbone with functional groups that will serve as anchor points for nanoparticle metal oxides. Nanoparticles such as PZT and PLZT, etc. must have active surfaces or functional groups that can bond with the polymer chain. The nanoparticles provide the piezoelectric function in the polymer and the backbone provides mechanical strength and structural integrity, electrical conductivity and other important properties. The backbone can be the same type of polymer as used for the structural layers. A new class of functional polymers have been synthesized at Pennsylvania State University, which can serve as the host for piezoelectric actuation.

\section{SAFETY AND RELIABILITY ISSUES}

There are many restrictions associated with RF technology that need to be considered. One of the main regulations is related to bandwidth and power dissipation. The relationship between the different frequency ranges and the maximum radiated power is shown in Table 1. The Australian Communications Authority (ACA) releases licences for companies and strictly governs bandwidth and power emission. One of the main restrictions is on interference - devices must not cause interference to other radio communications services and must operate within the relevant ISM bands. ${ }^{6}$

Table 1. This table shows the maximum radiated power that is allowed to be send through the human body at different frequency ranges. ${ }^{6}$

\begin{tabular}{|l|l|}
\hline Frequency Band $[\mathrm{MHz}]$ & Maximum Isotropically Radiated Power \\
\hline $915-928$ & $1 \mathrm{~W}$ \\
\hline $2400-2463$ & $4 \mathrm{~W}$ \\
\hline $2463-2483.5$ & $20 \mathrm{~mW}$ \\
\hline $5725-5875$ & $1 \mathrm{~W}$ \\
\hline
\end{tabular}

One of the fundamental concerns for RF in vivo applications is how the radiation affects the human body. Countless tests have been done researching a link between RF exposure and cancer. Hence a unit of measure called the Specific Absorption Rate (SAR) has been developed. The SAR is the basic unit of measurement of RF fields between $1 \mathrm{MHz}$ and $10 \mathrm{GHz}$, with a SAR of $4 \mathrm{~W} / \mathrm{kg}$ needed to produce adverse health effects. ${ }^{1}$ It has also been claimed that RF exposure induces heating in the body, which is thought to affect male fertility. ${ }^{1}$ 
This is an area that needs to be investigated further. The maximum SAR is dependent on where in the body the device is placed so each application will have to verify the device is within these limits.

Neither the electric field (E) nor the magnetic field $(\mathrm{H})$ penetrate far into a "good" conductor. The point where the fields are reduced by a factor of $1 / e \sim 1 / 2.71$ is called the electrical skin depth. Actuation forces (including external load) required for such a microvalve can be typically of the order of $100 \mu \mathrm{N}^{9}$ and moving through, say, a $10 \mu \mathrm{m}$ displacement, corresponds to $1 \mathrm{~nJ}$ of energy.

Using the formula for the electrical skin depth given by

$$
\delta=\sqrt{\frac{2}{\omega \sigma \mu}},
$$

where $\omega$ is the frequency in $\mathrm{rad} / \mathrm{s}, \mu$ the relative permeability and $\sigma$ the conductance we can see that for $f=900 \mathrm{MHz}$ we have a penetration depth of $4.2 \mathrm{~cm}$ into human muscle or $3.9 \mathrm{~cm}$ into human skin. ${ }^{10}$ That means that the antenna cannot be any further under the skin or muscle than these depths, if the signal is to be adequately received. It should be noted that the electrical skin depth is highly frequency and material dependent and hence will vary depending on the context in which it is used.

For our calculations we assumed the worst case of $100 \mathrm{~nJ}$ of actuation energy required by the valve. The SAW device aperture can oscillate in the $10 \mathrm{~Hz}-100 \mathrm{~Hz}$ range, so we arbitrarily pick $50 \mathrm{~Hz}$, for example. So the maximum delivered power of the antenna must be $20 \mu \mathrm{W}$ for the $50 \mathrm{~Hz}$ example. From this we can work out the minimum power requirements through the air using the Friis equation:

$$
P_{R}=P_{T} G_{T} G_{R}\left(\frac{\lambda}{4 \pi R}\right)^{2}
$$

Where $G$ is the gain, $R$ the distance and $P$ the power of each antenna. We must also consider the attenuation of the power through the human skin and check that this is within the regulations. We also calculated the transmission through skin, and the penetration depth, attenuation and surface power of radio frequency signals for skin and muscle at different frequencies. The results of those calculations are shown in Table 2.

\begin{tabular}{|l|l|l|l|l|l|l|l|}
\hline \multirow{2}{*}{$\begin{array}{l}\text { Frequency } \\
\mathrm{MHz}\end{array}$} & $\begin{array}{l}\text { Transmission } \\
\text { through skin }[\%]\end{array}$ & \multicolumn{2}{l|l|}{$\begin{array}{l}\text { Penetration depth } \\
\mathrm{m}\end{array}$} & \multicolumn{2}{l|}{$\begin{array}{l}\text { Attenuation } \\
\mathrm{dB}\end{array}$} & \multicolumn{2}{l|}{$\begin{array}{l}\text { Surface power } \\
\mu \mathrm{W}\end{array}$} \\
\cline { 3 - 8 } & & skin & muscle & skin & muscle & skin & muscle \\
\hline 434 & 26.9 & 0.0551 & 0.0525 & 0.119 & 0.128 & 76.1 & 75.45 \\
\hline 920 & 26.5 & 0.0396 & 0.0417 & 1.37 & 1.18 & 88.4 & 86.5 \\
\hline 2450 & 27.8 & 0.0224 & 0.0221 & 1.42 & 2.36 & 84.7 & 94.4 \\
\hline
\end{tabular}

Table 2. This table shows the transmission of radio waves through skin, their penetration depth, attenuation and surface power for skin and muscle at different frequencies. It shows that the penetration depth in skin and muscle decreases while with the increase of the frequency and that the attenuation of the body tissue and the surface power increase with increase of the used frequency.

The attenuation of the body tissue was calculated by the following equations:

$$
\begin{gathered}
A=e^{(-|\mathfrak{J}(\beta)| d)} \\
\beta=\frac{\omega}{c} \sqrt{1-\frac{j \sigma}{2 \omega \varepsilon}},
\end{gathered}
$$

where the attenuation is a term in solution of the wave equations, $\omega$ is the frequency in rads/s, $c$ is the velocity of light, $\sigma$ the conductivity and, $\varepsilon$ the permittivity of the medium. Because the conductivity and permittivity are highly frequency and material dependent, the values will vary depending on the context in which they are 
used. $^{11}$ We assumed $d=5 \mathrm{~cm}$ for the distance between the transmit and receive antennas.

Since $\frac{\sigma}{\omega \varepsilon}$ is small, we can use a Taylor approximation to give

$$
\beta=\omega / c\left(1-\frac{j \sigma}{2 \omega \varepsilon}\right)
$$

To make the relationship between the antenna size, width and efficiency at different frequencies clearer, we calculated the values shown in Table 4 by the following equations.

We calculated the efficiency by using the basic equation for short dipole antennas:

$$
\begin{gathered}
\eta=\frac{R_{\text {rad }}}{R_{\text {ohm }}+R_{\text {rad }}} \\
R_{\text {rad }}=80\left(\frac{\pi l}{\lambda}\right)^{2} \\
R_{o h m}=\frac{l R_{s}}{3 \pi a} \Rightarrow R_{s}=\frac{1}{\delta \sigma},
\end{gathered}
$$

where $l$ is $1 / 2$ length of the dipole, $a$ the radius of the antenna of $1 \mathrm{~mm}, R_{s}$ the surface resistance of the material and $\delta$ the electrical skin depth. We also assumed a gain of the transmitting antenna of 1.5 and $\sigma=4.1 \times 10^{7}$ $\mathrm{S} / \mathrm{m}$ for typical antenna metal (gold).

Initial research suggested the efficiency of the proposed antenna would be quite low and this would make the required transmit power exceed the regulations, which would make the construction of the devices untenable. However, after calculations it can be seen that the efficiency remains quite high, because the antenna thickness $(1 \mathrm{~mm})$ is reasonably large compared to the antenna length $(1 \mathrm{~cm})$. From this we can see that using an antenna of this size is a viable solution to meet the required specifications. To make that more clear we calculated the antenna size and length for different frequencies and also the efficiency and the transmitted power. The results of those calculations are shown in Table 3.

Table 3: This table shows sizes and properties of simple dipole antennas at different frequencies

\begin{tabular}{|l|l|l|l|l|l|l|l|}
\hline $\begin{array}{l}\text { Operating } \\
\text { frequency } \\
\mathrm{MHz}\end{array}$ & $\begin{array}{l}\text { Antenna } \\
\text { size } \\
\mathrm{cm}\end{array}$ & $\begin{array}{l}\text { Antenna } \\
\text { length } \\
\mathrm{cm}\end{array}$ & $\begin{array}{l}\text { Wave } \\
\text { length } \\
\mathrm{cm}\end{array}$ & $\begin{array}{l}\text { Efficiency } \\
\text { ( }\end{array}$ & $\begin{array}{l}\text { Far } \\
\text { field } \\
\mu \mathrm{m}\end{array}$ & $\begin{array}{l}\text { Transmitted } \\
\text { power } \\
\mu \mathrm{W}\end{array}$ & $\begin{array}{l}\text { Inside } \\
\text { regulations }\end{array}$ \\
\hline 434 & 1 & $\lambda / 69$ & 69 & 96 & 290 & 29.12 & $\mathrm{Y}$ \\
\hline 920 & 1 & $\lambda / 33$ & 33 & 99 & 606 & 147.5 & $\mathrm{Y}$ \\
\hline 2450 & 1 & $\lambda / 12$ & 12 & 99.8 & 1670 & 995 & $\mathrm{Y}$ \\
\hline & & & & & & & \\
\hline 434 & 0.5 & $\lambda / 138$ & 69 & 92 & 72 & 30.4 & $\mathrm{Y}$ \\
\hline 920 & 0.5 & $\lambda / 66$ & 33 & 97 & 151 & 146 & $\mathrm{Y}$ \\
\hline 2450 & 0.5 & $\lambda / 24$ & 12 & 99.9 & 417 & 994 & $\mathrm{Y}$ \\
\hline & & & & & & & \\
\hline 434 & 0.25 & $\lambda / 69$ & 69 & 86 & 19 & 32.6 & $\mathrm{Y}$ \\
\hline 920 & 0.25 & $\lambda / 69$ & 33 & 94 & 38 & 155.4 & $\mathrm{Y}$ \\
\hline 2450 & 0.25 & $\lambda / 69$ & 12 & 98 & 104 & 1014 & $\mathrm{Y}$ \\
\hline
\end{tabular}

There are several design techniques that may allow us to design an antenna that is small enough. Some of the most currently common methods include using a meander antenna. ${ }^{12}$ Further design methods include fractal antenna design, which is the process of minimizing the area of a square loop using fractal shapes. This allows the loop to be at least four times smaller than non fractal designs, for comparable efficiency. ${ }^{13} \mathrm{~A}$ fractal antenna approach also allows a smaller resonant frequency to be used compared with an ordinary approach. This 
means that a larger receiving area can be fitted into the same size, which increases the overall power received. ${ }^{14}$ One of the current methods for fractal approaches uses Hilbert curves. ${ }^{15}$ A comparison between a dipole and a Sierpinski fractal antenna is shown in Figure 4.

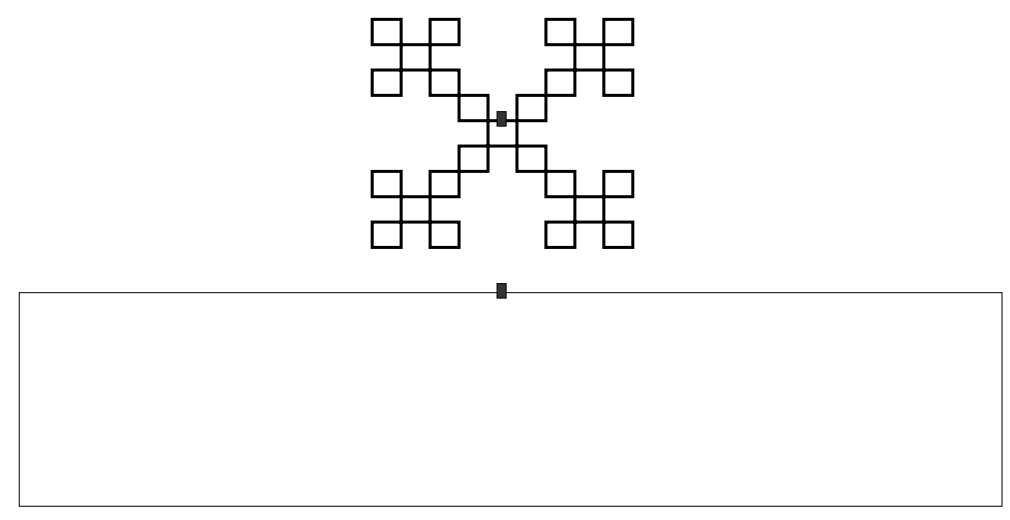

Figure 4. This figure shows a schematic comparison of two folded dipoles. The top one is an efficient Sierpinski fractal loop about $1 / 4$ the area of the loop (below), with virtually the same gain. ${ }^{13}$

\section{APPLICATIONS}

This section describes some of the main biomedical fields in which the RF controlled microvalve may be applied, i.e., 1) fertility control, 2) drug delivery, 3) flow cytometry and 4) DNA sequencing.

\subsection{Fertility Control}

There are a variety of methods for conception control such as using the "pill" or performing a vasectomy. But the choice of a suitable method is very difficult and the potential risks and benefits must be carefully analyzed. ${ }^{16}$ For example there are many side effects to using the "pill" and a vasectomy reversal has a high failure rate containing a painful surgical procedure with an inconvenient recovery time for the patient. ${ }^{17}$ To avoid these problems the microvalve described above could be used. It could be placed in the vas deferens of a male to make restored fertility possible and relatively easy. Within the first two weeks after insertion, the valve can be episodically switched on and off, reducing the backpressure during the early stages when the valve adheres to the walls of the vas deferens and to reduce any possible build up of proteins on the face of the valve. The insertion can be performed by a hypodermic needle. ${ }^{18}$

For the purposes of initial analysis of the problem, one can ignore geometric details, and examine for instance an oscillating aperture. However even that simplification poses a spectrum of possibilities between on the one hand a hole time-varying radius in a wall of negligible thickness and on the other a long pipe of time-varying radius through a very thick wall. These present completely different difficulties for fluid flow.

The decline of oscillatory flow-rate with frequency indicates that despite the small tube diameter, in the frequency range of interest the situation is far from being purely Stokes flow; momentum plays a highly significant role in determining the flow that occurs in response to the applied pressure gradient. In the present context, what is here determined is the extent of forward flow for a given frequency of opening orifice, relative to the flow that would occur through the tube if the microvalve presented no obstruction at all by periodic closure. It is also assumed that no partial obstruction of the tube occurs when the microvalve is open.

The fluid dynamic simulation has a typical low pass characteristic with a cut off frequency $f_{c}$ of $30 \mathrm{~Hz}$. The frequency range of interest here is between $10 \mathrm{~Hz}$ and $100 \mathrm{~Hz}$. In that particular range, the flow-rate relative to that at $0 \mathrm{~Hz}$ is $90 \%$ for $10 \mathrm{~Hz}$ and $25 \%$ for $100 \mathrm{~Hz}$. From the physiological point of view, the flow rate at $100 \mathrm{~Hz}$ is sufficient for the sperm transport through the microvalve, whereas it is desirable to have a higher flow-rate. The flow simulation shows that a material with special properties is needed that has an oscillation frequency in that range. Oscillations at such low frequencies are achieved by a special arrangement and distances of electrodes 
on the surface of the SAW device and by the fact that the nano-particle embedded polymer is a highly damped material. Frequencies down to $100 \mathrm{~Hz}$ have been achieved at Pennsylvania State University and work is ongoing for further reduction.

We are unable to insert the microvalve in the female ovarian duct because the ovarian duct has an inner surface which is coated with microvilli that are like microscopic fingers which help the ova to move along the duct in the correct direction. The ovarian duct is also very sensitive to foreign objects which tends to cause adhesion within the tube. ${ }^{18}$

\subsection{Drug Delivery}

In many different cases is it very useful, when drugs can be delivered at the right time and as close to the treatment area as possible, ${ }^{19}$ as for example, in the case of diabetes mellitus. To make this possible, drug delivery micropumps that can be implanted in the body are used to deliver small quantities of drug from a reservoir. These micropumps would avoid the strain on veins of weekly or daily injections. In this case, a RF controlled microvalve could be integrated in an implanted drug delivery system and make it possible to set the correct drug dosage to be applied at the right time. ${ }^{7}$ Existing approaches either use an osmotic valve, which unfortunately provides a continuous feed, or have the disadvantage of battery power.

\subsection{Flow Cytometry}

The basis of flow cytometry is that specific optical characteristics (such as fluorescence, light scatter) can provide a measure of the specific physical or chemical properties of biological particles (such as size and DNA content). The principle of flow cytometry has been integrated into a micromachined silica flow chamber. ${ }^{20}$ In this instrument a collection of small particles is pumped through a specially designed transparent tube. A measurement is made when the particles pass the "sensoring region". This region is delimited by the illumination and collection regions which are provided by light source and optical detector assemblies.

The RF controlled microvalve could be integrated in a flow cytometry system to control the stream of particles, which would keep the biological cells of interest in a separate particle chamber for later investigations. It could also prevent a reverse flow of the cells out of this chamber. ${ }^{7}$

\subsection{DNA Sequencing}

Present DNA sequencing methods employ electrophoresis and they are still quite slow and expensive. To speed up the sequencing process and reduce costs through miniaturization, one idea is to pull a strand of DNA through a tiny hole in a polymer material immersed in an ion solution. ${ }^{21}$ Variations of the ion current are detected, which correspond to the A, G, C or T bases in the DNA molecule. One key problem is that of poor signal-to-noise ratio (SNR) in the measured current. The proposed SAW-on-polymer devices provides a means to adaptively modulate the diameter of the hole to optimize the SNR, via use of lock-in amplifier techniques.

\section{OPEN QUESTIONS AND FUTURE WORK}

From the information provided we can see that there is a wide scope for future development. This development will be in several key areas, ranging from creating new polymers with more beneficial characteristics, through to investigating and designing new applications for the microvalve as a sensor and an actuator. One application that we think will be developed further is some form of heart monitoring device that could trigger and alarm if heart attack is imminent. In particular further research needs to be done in the calculation of input code length, i.e. to calculate the minimum length for safe operation of the device in in vivo applications. Another key issue that needs to be addressed is the size of the receiving antenna. By utilizing fractal or meander antenna designs the range of applications for the microvalve and SAW RFID should increase markedly so that it becomes common use in many areas of biomedicine. Future developments may use a gel of some form that is used in sensor applications to reduce reflection due to the change in impedance from skin to air.

For a given frequency, sub-optimal small antennas may be possible at the expense of increased external RF power - the limit to this trade-off will depend on the safety requirement for transcutaneous RF transmission for each given application. Given that the human vas deferens is of the order of $400 \mu \mathrm{m}$ in diameter, male 
fertility control is a rather ambitious goal, as the whole integrated valve would have to fit into that diameter. Therefore our near-term goal will be to firstly target drug-delivery applications, where the size restriction can be greatly eased. Size requirement will impact on the lowest usable RF frequency, and in this program we will push valve size and antenna size down as far as we can go to find the limits. The ultimate goal of a $400 \mu \mathrm{m}$ size for male fertility control is not untenable, however. To achieve this would require high a dielectric layer on a chiral absorber, using a fractal microantenna approach. Note also that the space saving by using the Sierpsinki fractal approach of Puente ${ }^{22}$ has recently been surpassed by using a Hilbert curve fractal. ${ }^{15}$

Another question is whether the SAW device can exert the forces required to control flow against ultimately physiological pressure gradients. In the short term, the proposed device will simply be limited to a narrow range of applications, if higher pressure gradients prove problematic. In the longer term, new polymer substrate materials may emerge (e.g. nanotube embedded polymer) that exhibit higher actuation strains.

The aim of material simulations is to find a material which needs a very low energy level to cause a relative high deformation. The frequency behavior of the material could be calculated by a simulation of the valve. Together with a simulation of the different shapes of microvalves, we can optimize microvalve set-up for fertility control. As a result of these simulations, a first model could be constructed following the required design specifications.

In modelling the SAW device, different acoustic modes need to be analyzed - for example, one would expect horizontal shear modes to be more useful than Rayleigh modes. This is because when a SAW device is in vitro Rayleigh modes are rapidly damped out. Horizontal mode polymer devices have been advised at Pennsylvania State University - these are manufactured by pulling the polymer melt through a field, so that the piezoelectric nanoparticles align in the preferred direction for horizontal mode propagation.

\section{CONCLUSION}

In this paper the investigation into future of RFID in biomedical applications was discussed. By using wireless communication and surface acoustic wave devices, implantable devices such as pacemakers, drug delivery pumps or microvalves could be controlled by a radio frequency signal without causing strain to a patient. By creating a surface acoustic wave on the surface of a polymer with piezoelectric properties, this polymer expands and contracts. Using this principle in biomedical applications would avoid the use of and batteries or wired connections, which decreases the overall size of the device and leads to a wider variety of possible applications such as vein valves.

We also discussed the encoding of SAW devices, which is very important for successful in vivo applications. We can achieve encoding of SAW devices by using the IDT's fingers in a specific order. Their polarity and orientation would be related to the input code and we could ensure that only the correct acoustic wave propagates. The maximum code length of reply is restricted at the moment to 32 bits.

We have determined that the efficiency of the proposed antenna is quite high, because the antenna thickness of $1 \mathrm{~mm}$ is reasonably large compared to the antenna length of $1 \mathrm{~cm}$. From this one can see that using an antenna of this size would be a viable solution to meet the required specifications.

The fluid dynamic behavior of the microvalve was discussed in the case study for the fertility control application, where the flow-rate through the oscillating valve aperture has a low-pass characteristic. The frequency range of interest is between $10 \mathrm{~Hz}$ and $100 \mathrm{~Hz}$. These frequencies are achieved by a special arrangement of surface electrodes on the actuator material of the valve and arranging suitable acoustic dispersal in the polymer. So far acoustic frequencies as low as $100 \mathrm{~Hz}$ have been achieved at Pennsylvania State University. The flow-rate of sperm through the microvalve still obeys physiological requirements for the fertilization of the human ovum in the frequency range of $10-100 \mathrm{~Hz}$. The flow-rate in this range relative to the flow-rate of $100 \%$ at $0 \mathrm{~Hz}$ is $75 \%$ at $100 \mathrm{~Hz}$ and only $10 \%$ at $10 \mathrm{~Hz}$.

\section{ACKNOWLEDGMENTS}

The authors would like to thank all members of both the Department of Electrical and Electronic Engineering and the Centre of Biomedical Engineering at the University of Adelaide, especially Leonard Hall and David Saint David Saint, as well as Hedley Hansen (DSTO), Chris Bertram (The University of New South Wales), Simon 
Maddocks (Department aof Animal Science, The University of Adelaide) and Stefan Enderling (The University of Edinburgh) for their helpful support.

\section{REFERENCES}

1. D. de Leeuw, "Plastic electronics," Physics World, March, pp. 31-34, 1999.

2. M.J. Madou, "Fundamentals of microfabrication," CRC Press, 1997.

3. M.J. Madou and M. Tierney, "Micro-Electrochemical valves and methods," US Patent, 5,368,704, 1994.

4. M.J. Madou and L.W. Low, "Microactuators towards microvalves for controlled drug delivery," Sensors and Actuators B: Chemical, 67(1-2), pp. 149-160, 2000.

5. F. Schmidt, O. Sczesney, C. Ruppel, and V. Magori, "Wireless interrogator system for SAW-identificationmarks, and SAW-sensor components," IEEE International Frequency Control Symposium, No. 96CH35935, pp. 208-215, 1996.

6. Australian Communications Authority http://www.aca.gov.au/publications/info/spreadsp.htm

7. S. Enderling, D. Abbott, V.K. Varadan, K.F. Boehringer, and Editors, "Directions for RF controlled intelligent microvalve," Smart Electronics and MEMS II Proceedings of SPIE Vol. 4236, pp. 204-212, 2001.

8. V.V. Varadan, "IDT sensors for detection of ice on rotorcraft," Proc. SPIE Smart Electronics and MEMS, Vol. 3328, San Diego, pp. 49-58, March 1998.

9. M. Kohl, J. Gottert, and J. Mohr, "Verification of the micromechanical characteristics of linear actuators," Sensors and Actuators, Vol. 53, pp. 416-422, 1996.

10. C. Gabriel, "Compilation of the dielectric proporties of body tissues at RF and microwave frequencies," Report N.AL/OE-TR-1996-0037, Occupational and Environmental Health Directorate, Radiofrequency Radiation Division, Brooks Air Force Base, Texas(USA), 1996.

11. Italian National Research Council, Institutes of Applied Physics http:niremf.iroe.fi.cnr.ittissprophtmlcliehtmlclie.htm

12. V.K. Varadan, P.T. Teo, K.A. Jose, and V.V. Varadan, "Design and development of a smart wireless system for passive temperature sensors," Smart Materials and Structure, pp. 379-378, 2000.

13. T. Vernon, "Fractal antennas offer benefits," Radio World, September, 1999.

14. V.K.Varadan, K.J. Vinoy, and V.V. Varadan, "RF MEMS and reconfigurable conformal fractal antennas," Smart Electronics and MEMS, Proceedings of SPIE Vol. 4236, pp. 194-202, 2001.

15. K.J. Vinoy, K.A. Jose, V.K. Varadan, and V.V. Varadan, "Hilbert curve fractal antenna: a small resonant antenna for VHF/UHF applications," Microwave and Optical Technology Letters, Vol. 29, No. 4, pp. 215-19, May 2001.

16. F. Martini, Fundamentals of Anatomy and Physiology, ch. 21 and 28, Prentice Hall, Englewood Cliffs, New Jersey, 2nd ed., pp. 669,950, 1992.

17. H. Fisch, "The patient's guide to vasectomy reversal," http://www.cpmcnet.columbia.edu/dept/urology/vasrev.html

18. D. Moffett, Human Physiology: Foundations and Frontiers, ch. 24, Mosby, 2 ed., page 688, 1993.

19. P. Dario, M.C. Carrozza, A. Benevenuto, and A. Menciassi, "Microsystems in biomedical applications," Journal of Micromechanical Microengineering Vol. 10, pp. 235-244, 2000.

20. D. Sobek, S.D. Senturia, and M.L. Gray, "Microfabricated fused silica flow chambers for flow cytometry," Proceeding of Solid-State Sensors and Actuators Workshop, pp. 260-263, June 1994.

21. J. Kasianowicz, E. Brandin, D.W. Branton, and D. Deamer, "Characterization of individual polynucleotide molecules using a membrane channel," Proc. Natl. Acad. Sci., USA, 93(24), pp. 13770-13773, 1996.

22. C. Puente, J. Anguera, C. Borja, and J. Soler, "Fractal-shaped antennas and their application to GSM 900/1800," J. Inst. British Telecom. Eng., Vol. 2, Pt. 3, pp. 92-95, July-September, 2001. 